\title{
Transmission of multidrug-resistant and extensively drug-resistant tuberculosis in a metropolitan city
}

\author{
Eric Chung Ching Leung*, Chi Chiu Leung*, Kai Man Kam*, Wing Wai Yew", \\ Kwok Chiu Chang*, Wai Man Leung* and Cheuk Ming Tam*
}

ABSTRACT: Multidrug-resistant (MDR)- tuberculosis (TB) and extensively drug resistant (XDR)TB reportedly lead to increased household transmission.

This is a retrospective cohort study of active TB occurring among household contacts exposed to MDR-TB.

Of 704 contacts in 246 households, initial screening identified 12 (1.7\%) TB cases (prevalent cases) and $17(2.4 \%)$ contacts that subsequently developed active TB (secondary cases) after a median (range) duration of 17 (5-62.5) months. Eight prevalent cases and three secondary cases had MDR-TB. TB incidence rates per 100000 person-years were 254.9 overall and 45.0 for MDRTB. XDR-TB in the index MDR-TB patient significantly increased the odds of identifying a prevalent TB case to 4.8 (95\% $\mathrm{Cl} 1.02-22.5)$, and the hazard of finding a secondary TB case to $4.7(95 \% \mathrm{CI}$ 1.7-13.5). Molecular fingerprinting confirmed household transmission of MDR-TB. Of 20 retrievable isolates from 27 XDR-TB index cases, restriction fragment length polymorphism analysis showed clustering among 13 (65\%), with 11 (55\%) due to recent transmission by $n-1$ method and an identifiable household source in only three (27.2\%) of the 11 cases.

XDR-TB relative to MDR-TB significantly increases household transmission of TB, probably reflecting prolonged/higher infectivity, and indicating a need for prolonged household surveillance. XDR-TB may largely transmit outside of the household settings.

KEYWORDS: Case finding, contact investigations, extensively drug-resistant tuberculosis, multidrug-resistant tuberculosis

$\mathbf{T}$ uberculosis (TB) is still an infectious disease of public health importance today. Of particular concern is the occurrence of multidrug-resistant (MDR)-TB defined by bacillary resistance to at least isoniazid and rifampicin, and extensively drug-resistant (XDR)-TB, which is MDR-TB with additional resistance to any fluoroquinolone, and at least one of the three secondline injectable drugs (kanamycin, amikacin and capreomycin). MDR-TB and XDR-TB reportedly require prolonged treatment, with a high risk of treatment failure $[1,2]$. Slow bacteriological conversion and treatment failure may increase the risk of transmission within the community, as suggested by reports of secondary TB transmission within the household [3, 4]. However, few studies have systematically evaluated the magnitude of such transmission risks within a defined geographical area, such as a metropolitan city.

Designated as a Special Administrative Region in the People's Republic of China, with autonomy regarding its own infrastructure including housing, transport and healthcare, Hong Kong is a metropolis with a population of 7 million. It is classified by the World Health Organization (WHO) as an intermediate TB burden area. The annual incidence and notification rate of TB cases in Hong Kong has steadily declined from 7072 cases (109 per 100000 population) in 1997 to 5193 cases (74 per 100000 population) in 2009 [5]. Around 1\% of the cultureconfirmed TB cases are MDR-TB (30-40 cases each year), of which approximately $10 \%$ are XDR-TB [5]. Around $40-50 \%$ of the MDR-TB (15-20 cases) cases are new cases without history of previous $\mathrm{TB}$ treatment $[6,7]$. It has been a regular practice in Hong Kong to screen for active TB among household TB contacts regardless of sputum smear status [8]. Chest radiography (CXR) is the main screening tool. All contacts with initially negative screens are advised to seek immediate medical attention for any subsequent symptoms and signs suggestive of TB. Further workup is carried out in the presence of CXR abnormalities or suspicious symptoms.
AFFILIATIONS

*Tuberculosis and Chest Service, Centre for Health Protection, Dept of Health, Hong Kong,

"Tuberculosis Reference Laboratory, Public Health Laboratory Centre, Centre for Health Protection, Hong Kong, and

'Department of Microbiology, The Chinese University of Hong Kong, Hong Kong, Hong Kong SAR, China.

\section{CORRESPONDENCE}

Eric Chung Ching Leung

Wan Chai Chest Clinic

99 Kennedy Road

Wan Chai

Hong Kong SAR

China

E-mail: eric_leung@dh.gov.hk

Received:

May 042012

Accepted after revision:

June 272012

First published online:

Aug 092012

European Respiratory Journal Print ISSN 0903-1936 Online ISSN 1399-3003 
Because of universal neonatal bacille Calmette-Guérin vaccination coverage (and extensive revaccination in primary schools before 2000) [9], the tuberculin skin test was not used routinely in the investigation of contacts except among young child contacts (aged below 5 years) of smear-positive index cases prior to 2005. As there is no universally agreed prophylactic treatment for MDR-TB, close contacts of MDR-TB cases are followed up halfyearly for active disease without any preventive treatment. The current study is a retrospective cohort analysis of household contacts exposed to MDR-TB in this locality.

\section{METHODS}

All MDR-TB patients notified from 1997 to 2006 were identified from the territory-wide TB registry and laboratory surveillance data as reported previously [10].

The contact investigation records of all MDR-TB patients (index cases) were reviewed to identify all household contacts, defined as persons living and sleeping in the same household as the index case for at least 1 month. Their initial screening and subsequent follow-up results were also ascertained from the contact investigation records. At the end of the study, the names and identification card numbers of all identified contacts were cross-matched with the territory-wide TB registry and death registry to detect any cases of TB or date of death after the active surveillance period. An observation was censored when the contact died from any causes other than TB or no TB developed by the end of the study period (31 May 2011). The rate of TB development was calculated as the number of active TB cases per person-years. Diagnostic and clinical information of all identified TB cases was verified by checking the relevant medical records. An active case of TB was defined as disease proven by isolation of Mycobacterium tuberculosis, or in the absence of bacteriological confirmation, disease diagnosed on clinical, radiological and/or histological grounds together with an appropriate response to TB treatment.

The respective relationships between the occurrence of prevalent and secondary TB cases among household contacts exposed to MDR-TB and the index case's characteristics were examined. A prevalent case was defined as a case of TB disease detected in the initial screening, whereas a secondary case referred to a case detected in the subsequent surveillance period. Univariate analysis of categorical data was performed by the Pearson's Chi-squared test or the Fisher's exact test, as appropriate. Significant predictors of prevalent and secondary TB cases were identified in logistic risk models and Cox models, respectively. A two-tailed $\mathrm{p}$-value $<0.05$ was taken as statistically significant. Statistical analyses were performed using SPSS version 16.

As part of the local surveillance, DNA fingerprinting with restriction fragment length polymorphism (RFLP) analysis was carried out in: 1) MDR-TB with a prevalent and secondary case identified in the contact screening to ascertain whether the same Mycobacterium tuberculosis strain was transmitted within the household; 2) All XDR-TB cases in this cohort to assess the degree of molecular clustering; using standardised methods with a 245-bp right-sided probe and internal molecular weight standards [11]. Clusters were defined as groups of two or more isolates having an identical IS6110 RFLP pattern, allowing for the presence, absence or shifting of no more than one band.
This study was approved by the ethics committee of the Department of Health (Hong Kong SAR, China). All patients were informed at the time of care delivery that the clinical data collected would be used for statistical analysis and research purposes.

\section{RESULTS}

Table 1 summarises the characteristics of the 270 MDR-TB cases with case categories and clinical outcomes classified according to the criteria proposed by LASERSON et al. [12]. Over $45 \%$ were new cases with no prior history of $\mathrm{TB}$ treatment (Laserson's MDR case category 1). Approximately 13\% were new immigrants. A total of $142(51.8 \%)$ were ever smokers. Comorbidities were present in 141 (52.2\%). Voluntary HIV testing was offered to all TB patients and the overall incidence of HIV positivity was low (1.1\%). Eight $(2.9 \%)$ cases showed potential epidemiological linkage by household (four families), including two pairs $(1.5 \%)$ of XDR-TB cases and two pairs $(1.5 \%)$ of other MDR-TB cases.

Figure 1 shows the flowchart of household contact tracing. Excluding 14 MDR-TB patients with no household contacts and 10 with missing contact records, 246 index MDR-TB patients were recruited for household contact analysis. Among 736 household contacts, 32 were excluded as they did not attend for screening. Among the 704 household contacts included in the final analysis, $12(1.7 \%)$ prevalent cases were found: three XDRTB cases associated with three XDR-TB index cases; one XDRTB, four MDR-TB, three drug-susceptible TB and one culture negative TB case associated with seven MDR-TB index cases. The median number of contacts per index case was three (range $1-8)$. RFLP analysis among 11 index-prevalent contact pairs showed $100 \%$ concordance in three pairs with XDR-TB, 100\% concordance in one MDR-TB index case pairing with one contact with XDR-TB, 50\% concordance in four non-XDR MDRTB pairs, and no concordance in three MDR-TB index cases pairing with three contacts with drug-susceptible TB.

Among the 692 household contacts with no TB found during the initial screening, $17(2.5 \%)$ secondary cases were found in 15 households, including six (two XDR-TB, two drug-susceptible TB and two culture-negative TB) cases among 94 contacts exposed to XDR-TB, and 11 (one MDR-TB, two streptomycin and isoniazid-resistant $\mathrm{TB}$, two drug-susceptible $\mathrm{TB}$ and six culture-negative TB) among 598 contacts exposed to non-XDR MDR-TB. RFLP among eight index-secondary contact pairs showed $100 \%$ concordance in one pair of XDR-TB patients, $100 \%$ concordance in one pair of non-XDR MDR-TB patients, $100 \%$ concordance in two MDR-TB index cases pairing with two contacts with non-MDR-TB (streptomycin and isoniazidresistant), and no concordance in four MDR-TB index cases pairing with four contacts with drug-susceptible TB. Both index cases of the two pairs of MDR-TB matching with nonMDR-TB were retreatment cases. The follow-up time was 6669 (mean \pm SD $8.77 \pm 3.98)$ person-years overall, 1005 (10.58 \pm 4.23$)$ person-years among contacts exposed to XDR-TB and 5664 $(8.51 \pm 3.88)$ person-years among contacts exposed to non-XDR MDR-TB, giving TB incidence rates of 254.9, 597.0, and 194.2 cases per 100000 person-years, respectively.

Tables 2 and 3 show the respective relationships between index case's characteristics and the occurrence of prevalent TB 


\begin{tabular}{|c|c|c|c|c|}
\hline $\begin{array}{l}\text { TABLE } 1 \text { Baseline characteristics of a cohort } \\
\text { 2006) }\end{array}$ & lltidrug-re & ADR) tuberculc & atients in & g (1997- \\
\hline Parameters & XDR-TB & Other MDR-TB & Total & p-value \\
\hline Subjects n & 27 & 243 & 270 & \\
\hline \multicolumn{5}{|l|}{ Demographics } \\
\hline Age years & $41(32-54)$ & $46(35-62)$ & $46(34-60)$ & 0.480 \\
\hline Nonpermanent immigrants ( $<7$ years) & $5(18.5)$ & $31(12.8)$ & $36(13.3)$ & 0.400 \\
\hline Ever smoker & $14(51.8)$ & 128 (52.6) & $142(51.8)$ & 0.670 \\
\hline $\begin{array}{l}\text { Adverse social factors (incarceration, drug addict, financial } \\
\text { assistance) }\end{array}$ & $7(25.9)$ & $64(26.3)$ & $71(26.3)$ & 0.963 \\
\hline \multicolumn{5}{|l|}{ Comorbidities } \\
\hline Diabetes mellitus & $2(7.4)$ & $45(18.5)$ & $47(17.4)$ & 0.149 \\
\hline Elderly care home or institutional & $0(0)$ & $8(3.3)$ & $8(3.0)$ & 0.339 \\
\hline CVS disease (HT, IHD) & $1(3.7)$ & $16(6.6)$ & $17(6.3)$ & 0.401 \\
\hline Liver cirrhosis & $0(0)$ & $7(2.9)$ & $7(2.6)$ & 0.372 \\
\hline Any comorbidity & $9(33.3)$ & $132(54.3)$ & $141(52.2)$ & 0.044 \\
\hline \multicolumn{5}{|l|}{ WHO case category } \\
\hline 1 (no history of TB treatment) & $11(40.7)$ & $113(46.5)$ & $124(45.9)$ & 0.001 \\
\hline 2 (treatment with first line for $>1$ month) & $10(37.0)$ & $124(51.0)$ & $134(49.6)$ & \\
\hline 3 (treatment of second line for $>1$ month) & $5(18.5)$ & $3(1.2)$ & $6(2.2)$ & \\
\hline 4 (transferred in cases) & $1(3.7)$ & $3(11.1)$ & $6(2.2)$ & \\
\hline Pulmonary TB only & $26(96.3)$ & $219(90.1)$ & $245(90.7)$ & 0.537 \\
\hline Pulmonary and extrapulmonary TB & $1(3.7)$ & $18(7.4)$ & $19(7.0)$ & \\
\hline Cavitary disease & $6(23.1)$ & $104(43.9)$ & $110(41.8)$ & 0.041 \\
\hline
\end{tabular}

Data are presented as median (range) or $\mathrm{n}(\%)$, unless otherwise stated. XDR: extensively drug resistant; CVS: cardiovascular disease; HT: hypertension; IHD: ischaemic heart disease; WHO: World Health Organization; AFB: acid-fast bacilli.

cases and secondary TB cases among household contacts exposed to MDR-TB. Among 607 household contacts exposed to 220 non-XDR MDR-TB index patients, nine prevalent TB cases (including one XDR-TB and four MDR-TB cases) and 11 secondary cases (including one MDR-TB case) were found; whereas among 97 household contacts exposed to 26 XDR-TB index patients, three prevalent TB cases (all XDR-TB cases) and six secondary cases (including two XDR-TB cases) were identified. Logistic regression analyses showed that sputum smear negativity and XDR-TB in the index case significantly increased the odds of identifying prevalent TB cases among household contacts exposed to MDR-TB to 4.3 and 4.8, respectively. Cox regression analyses showed that cavitary lung disease and XDR-TB in the index case significantly increased the hazards of having secondary cases among household contacts exposed to MDR-TB to 3.7 and 4.7 respectively.
The above regression analyses on prevalent and secondary cases were based on all the positive cases identified by contact tracing and not on the molecularly concordant cases, as some of the contact cases were culture-negative and not all of the positive cultures were retrievable for RFLP analysis. RFLP analysis performed in 11 retrievable isolates (eight drugresistant and three fully susceptible) among 12 prevalent cases and eight retrievable isolates (four drug-resistant and four fully susceptible) among 17 secondary cases in household contact tracing showed concordant cases in three XDR-TB clusters A, B and C; one XDR-TB to MDR-TB cluster D; two MDR-TB clusters E and G; and two MDR-TB pairing with streptomycin and isoniazid-resistant $\mathrm{TB}$ clusters $\mathrm{F}$ and $\mathrm{H}$ (table 4). In sensitivity analyses using prevalent and secondary cases identified to be "truly positive" by RFLP, XDR-TB was still shown to be a significant predictor for prevalent cases 
TABLE 2 Index case characteristics and the occurrence of prevalent tuberculosis (TB) cases among household contacts exposed to multidrug-resistant (MDR)-TB: logistic regression analyses

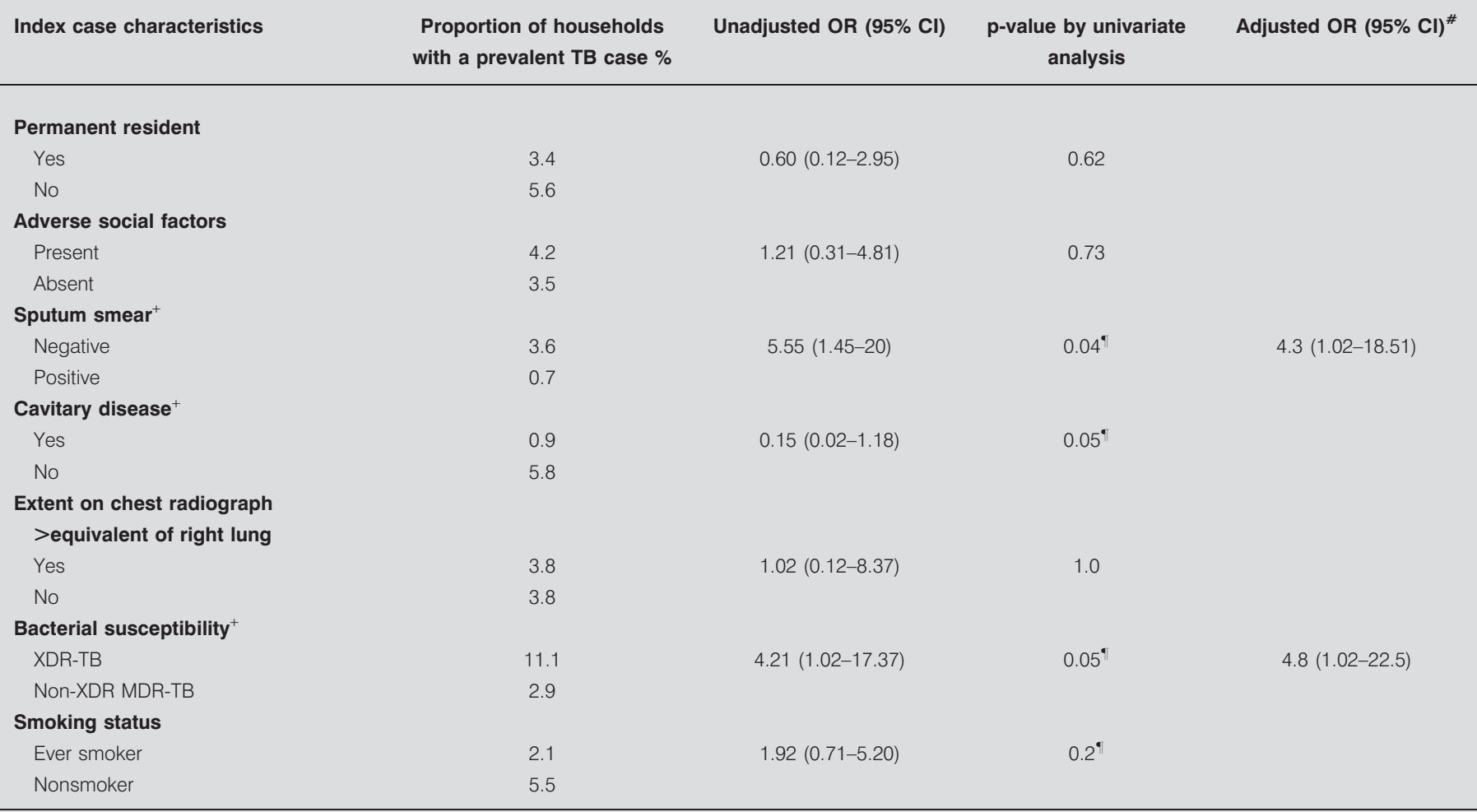

XDR: extensively drug resistant. ${ }^{\#}$ : variables were selected by backward stepwise selection with p-values for entry and removal being 0.05 and 0.10 , respectively; ${ }^{\circ}:$ values by Fisher exact test; ${ }^{+}$: covariates included in multiple logistic regression analysis.

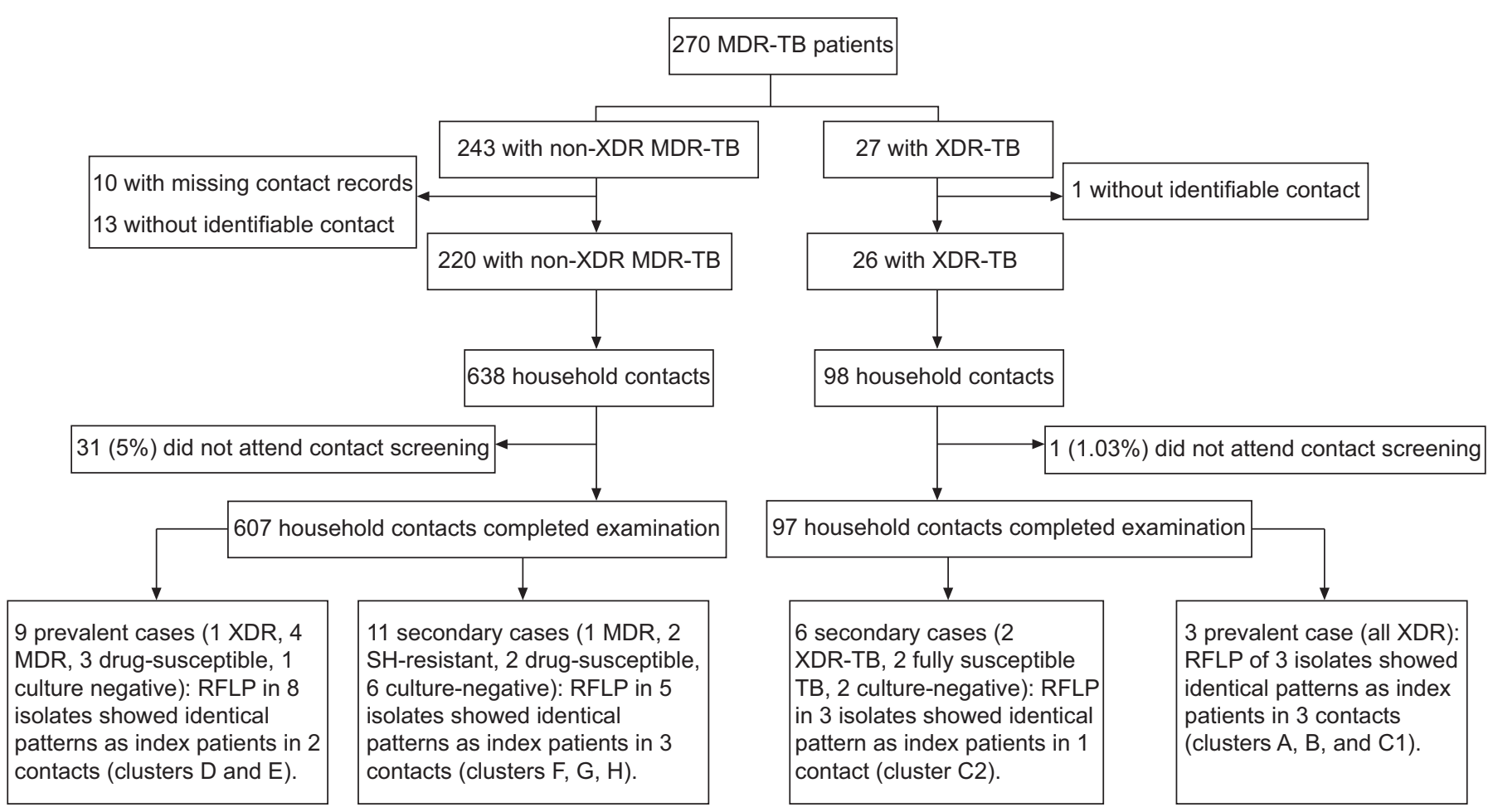

FIGURE 1. Contacts exposed to 270 multidrug-resistant (MDR) tuberculosis (TB) patients (1997-2006). XDR: extensively drug resistant; RFLP: restriction fragment length polymorphism; SH-resistant: streptomycin and isoniazid resistant. 


\section{TABLE 3 Index case characteristics and the occurrence of secondary tuberculosis (TB) cases among household contacts exposed to multidrug-resistant TB: Cox regression analyses}

\begin{tabular}{|c|c|c|c|}
\hline Index case characteristic & $\begin{array}{l}\text { Unadjusted hazard ratio } \\
\qquad(95 \% \mathrm{Cl})\end{array}$ & $\begin{array}{l}\text { p-value by univariate } \\
\text { analysis }\end{array}$ & $\begin{array}{l}\text { Adjusted hazard ratio } \\
\qquad(95 \% \mathrm{Cl})^{\#}\end{array}$ \\
\hline Permanent resident & $2.70(0.36-20.40)$ & 0.33 & \\
\hline Adverse social factors & $2.07(0.80-5.38)$ & 0.13 & \\
\hline XDR-TB' & $3.30(1.24-8.92)$ & 0.02 & $4.7(1.7-13.5)$ \\
\hline Cavitary disease & $2.68(1.02-7.05)$ & 0.05 & $3.7(1.4-10.3)$ \\
\hline Ever smoker & $1.92(0.71-5.20)$ & 0.20 & \\
\hline $\begin{array}{l}\text { WHO treatment outcome at } 2 \text { years: cured or } \\
\text { completed treatment }\end{array}$ & $0.48(0.18-1.26)$ & 0.14 & \\
\hline
\end{tabular}

$(\mathrm{p}=0.013)$ and a suggestive trend of association was also found for secondary cases $(\mathrm{p}=0.068)$.

RFLP analysis in 20 non-duplicate retrievable isolates from 27 XDR-TB cases included in this retrospective cohort showed nine additional cases outside the household in cluster A (eight cases) and cluster C (one case). Overall, molecular clustering was found in $13(65 \%)$ of 20 retrievable XDR-TB specimens (nine cases in cluster $\mathrm{A}$ and four cases in cluster C; $75 \%$ for new cases, $58.3 \%$ for retreatment cases). Disregarding the effect of incomplete sampling [13] and using the n-1 method [14] (cases other than the first case in each cluster are counted as resulting from recent transmission), 11 out of 20 (55\%) of XDR-TB cases were considered to have resulted from recent transmission, but only three (one case in cluster A and two cases in cluster C) or $27.2 \%$ of the above 11 cases had an identifiable source within the household. Figure 2 shows the temporal development of four XDR-TB molecular clusters (including one non-XDR MDR-TB case in cluster D).

\section{DISCUSSION}

In this study, 124 (45.9\%) of the MDR-TB cases notified in Hong Kong from 1997 to 2006 did not have prior history of anti-TB treatment. RFLP analysis confirmed household transmission of MDR-TB. A high degree $(65 \%)$ of molecular clustering was found among XDR-TB isolates, highlighting the importance of recent transmission ( $55 \%$ by $n-1$ method) with approximately a quarter of such transmission occurring within the household. $12(1.7 \%)$ prevalent TB cases were found among household contacts, with approximately two-thirds being MDRTB cases. $18(2.5 \%)$ secondary TB cases were found in the remaining household contacts cases occuring at a secondary TB rate of 255 per 100000 person-years with a mixture of drug resistance patterns. The occurrence of prevalent and secondary TB cases within a household can be independently predicted by the index case's characteristics: sputum smear negativity and XDR-TB for a prevalent TB case, and cavitary lung disease and XDR-TB for secondary TB cases.

Drug-resistant TB is either primary or acquired. Primary drug resistance occurs as a result of transmission of drug-resistant
TB strains whereas acquired drug resistance develops because of inadequate treatment. Traditionally, acquired drug resistance has been emphasised as the principal mechanism by which drug-resistant TB develops. However, in recent MDRTB reports, the prevalence of new cases with no previous TB treatment has been rising in both high-prevalence HIV settings (South Africa: 55\% [15]) and low-prevalence HIV settings (Shanghai, China: 60\% [16]; Latvia: 37\% [17]; Japan: 45\% [18]). In the present study, we found a similarly high proportion $(>45 \%)$ of new cases among MDR-TB patients (i.e. Laserson's MDR case category 1), and observed a much higher molecular clustering rate among the XDR-TB cases in this study $(65 \%)$ than the clustering rate reported among general TB patients (29.2\%) in Hong Kong [19]. Similarly high clustering rates were also reported among XDR-TB patients in Japan (71\%) [18] and Europe (82\%) [20], again highlighting the importance of recent transmission for these extensively drug-resistant cases. A substantial proportion of MDR-TB with primary drug resistance in Hong Kong may be anticipated from the significant decline in acquired drug resistance among MDR-TB cases [21]

Our study showed prevalent TB and incident TB in $1.7 \%$ and $2.5 \%$ of household contacts respectively. Overall, $\mathrm{TB}$, either incident or prevalent, is found in $4.1 \%$ of the MDR-TB contacts, which is higher than the corresponding prevalence rates of $1.9 \%$ and $1.7 \%$ reported among household contacts of drug-susceptible TB in the same locality $[8,22]$, but lower than the reported rates of 5\% in another MDR-TB contact study in 2011 [23] and $4.5 \%$ in one meta-analysis [24]. While smear positivity and cavitary disease were shown to be significant predictors of TB among contacts exposed to predominantly drug-susceptible TB in our previous study [22], this study found a significant association between an XDR-TB index patient and the occurrence of both prevalent and secondary cases among contacts exposed to MDR-TB. This likely reflects the prolonged duration of infectivity of index cases with XDR-TB. Heightened awareness of TB symptoms (e.g. chronic cough) in the presence of a known prevalent TB case in the household might have led to early medical consultation, and this may underline the association between the negative smear status of the index case with a prevalent case in the same household. 


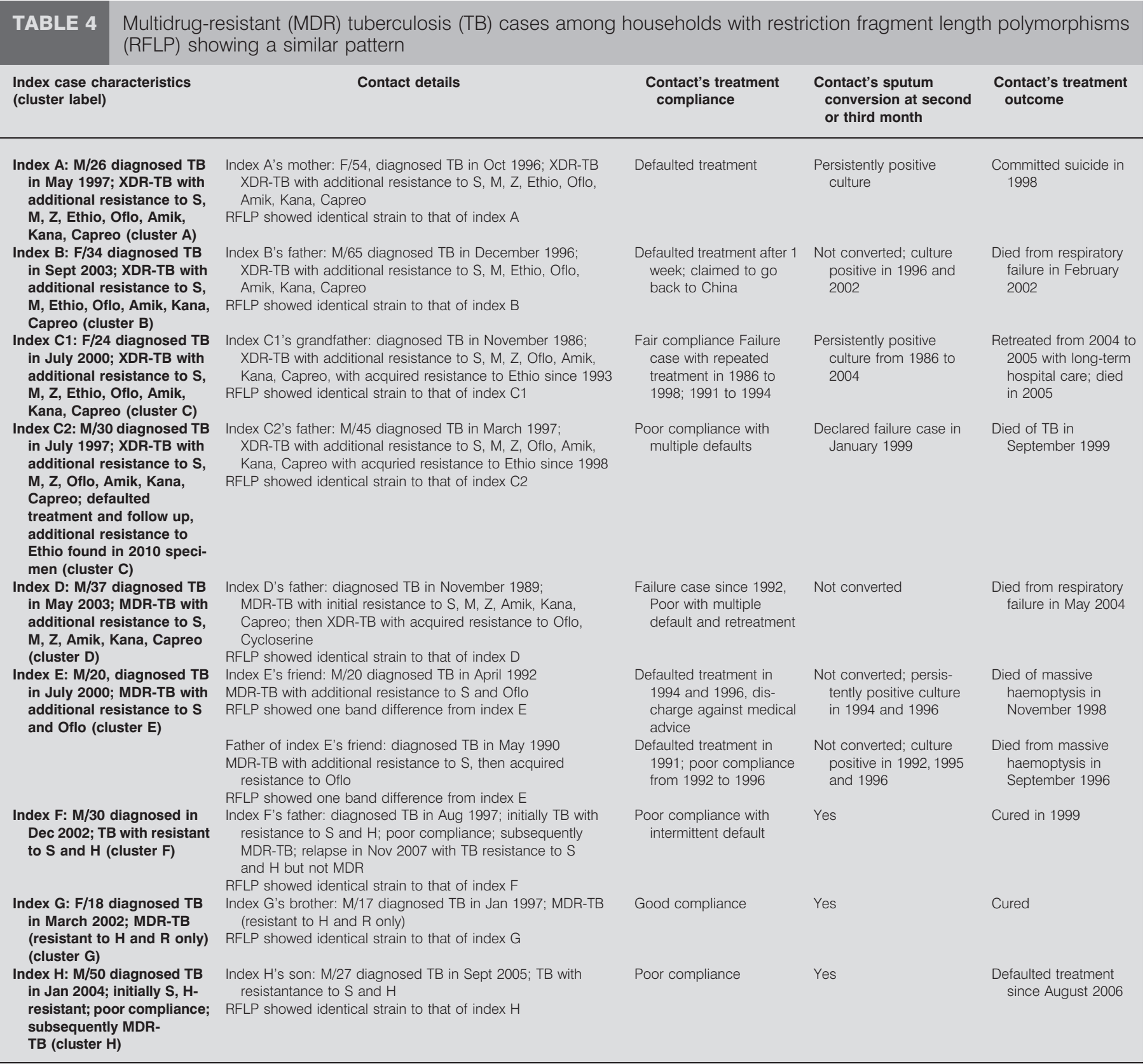

XDR: extensively drug resistant; S: streptomycin; H: isoniazid; R: rifampicin; M: ethambutol; Z: pyrazinamide; Ethio: ethionamide; Oflo: ofloxacin; Amik: Amikacin; Kana: Kanamycin; Capreo: capreomycin.

Among eight retrievable isolates from 17 secondary cases identified by the contact tracing, only four cases had concordant strains as proven by RFLP (XDR-TB cluster C2, MDR-TB cluster $\mathrm{G}$ and two MDR-TB pairing with streptomycin and isoniazidresistant $\mathrm{TB}$ clusters $\mathrm{F}$ and $\mathrm{H}$ ). The occurrence of different resistance patterns within individual molecular clusters could have resulted from progressive acquisition of resistance by the source strain during the prolonged infectious period while under treatment or the presence of multiple clones (of the same strain) showing varying degrees of drug resistance in the infected host.

RFLP analysis confirmed the transmission of MDR-TB among household contacts while regression analysis showed XDR-TB had a higher risk of household transmission among all MDRTB cases. On further examination of the clinical details in these households (table 4), most of them involved XDR-TB patients with persistent sputum culture positivity because of either default or treatment failure. Although the overall prognosis of XDR-TB patients is poor, with slow sputum culture conversion, high percentage of treatment failure and early mortality within 1 year $[1,2,15]$, some XDR-TB patients can survive for years (as in the case of Index C1's grandfather), thus posing a substantial risk to their families.

In this study, approximately a quarter of the presumably recently transmitted XDR-TB cases had traceable sources within 

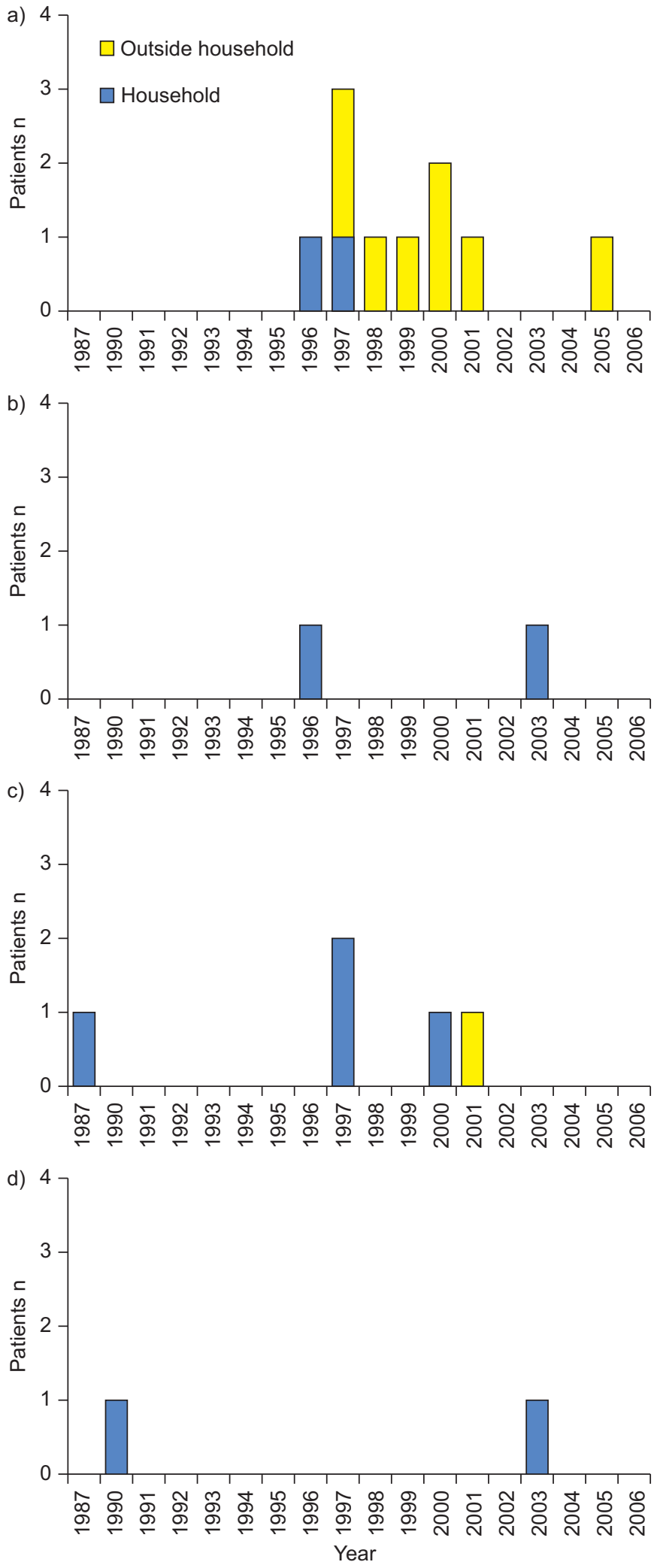

FIGURE 2. Temporal developments of four clusters (with the prevalent case in contact tracing included). a) Cluster A: 10 extensively drug-resistant (XDR) tuberculosis (TB) cases; b) cluster B: two XDR-TB cases; C) cluster C: five XDR-TB cases; and d) cluster $\mathrm{D}$ : one multidrug-resistant $T B$ and one XDR-TB case. the household. A major proportion of the ongoing transmission therefore appears to take place outside the household. Hong Kong, with a population of 7 million living in an area of $1104 \mathrm{~km}^{2}$, is one of the most densely populated regions in the world. It is conceivable that the congregated effect of the small transmission risks during very frequent person-to-person contact may have accounted for the high proportion of the molecular clustered cases without apparent epidemiological links. In Norway, an XDR-TB patient lost to follow-up led to an outbreak of 15 XDR-TB cases with the same strain with identical RFLP and spoligotyping DNA patterns, and this accounted for $40 \%$ of the country's MDR-TB cases from 1994 to 2005, highlighting the transmission risk of such cases in the community $[25,26]$. It has been suggested that evolution of drug resistance bears a fitness cost, thereby attenuating the overall transmissibility and virulence of the pathogen [27]. This premise might not hold true for some drug-resistant $M$. tuberculosis strains, e.g. those with a Ser315Thr mutation in the kat G gene, which has been recently reported with clustering of MDR-TB [28] and XDR-TB patients [18]. Recent studies have also shown that compensatory mutations in the isoniazid [29] and rifampicin [30] resistance gene conferred high competitive fitness both in vitro and in vivo, favouring the spread of these resistant strains.

In recent years, experts in high-prevalence drug-resistant TB settings have advocated long-term treatment of failure MDRTB patients in sanatoria to minimise infectious risk [31]. However, in the absence of effective treatment, strict isolation may be difficult to enforce, as it could imply life-long solitary confinement. Caution may also be required to minimise untoward publicity, which could adversely affect passive case finding, and unidentified drug-resistant cases could pose a greater risk of transmission within the community. In this regard, the WHO has expressed the view that forced isolation of drug-resistant TB should be used only as a last resort when other means have failed [32]. These pros and cons of setting up a sanatorium for drug-resistant TB should be shared with the general public and the final consensus should be based on the value judgment made by society.

In conclusion, the high proportion of new cases and molecular clusters among MDR-TB patients notified in Hong Kong highlighted the contribution of primary drug resistance within the densely populated community, which paralleled a falling trend in acquired drug resistance among MDR-TB patients. The occurrence of XDR-TB relative to non-XDR MDR-TB in the index patient significantly increases the risk of household transmission of TB. Nonetheless, this study suggests that XDR$\mathrm{TB}$ in Hong Kong is largely transmitted among lower-risk social contacts outside the household setting.

\section{STATEMENT OF INTEREST}

None declared.

\section{ACKNOWLEDGEMENTS}

We are grateful to the frontline staff of the Tuberculosis and Chest Service, Centre for Health Protection, Department of Health, for assistance in contact tracing; and the Clinical and Microbiological staff of Tuberculosis Reference Laboratory, Public Health Laboratory Centre, for performing the RFLP assays and other microbiological diagnostic procedures. 


\section{REFERENCES}

1 Mitnick CD, Shin SS, Seung KJ, et al. Comprehensive treatment of extensively drug-resistant tuberculosis. N Engl J Med 2008; 359: 563-574.

2 Keshavjee S, Gelmanova IY, Farmer PE, et al. Treatment of extensively drug-resistant tuberculosis in Tomsk, Russia: a retrospective cohort study. Lancet 2008; 372: 1403-1409.

3 Grandjean L, Crossa A, Gilman RH, et al. Tuberculosis in household contacts of multidrug-resistant tuberculosis patients. Int J Tuberc Lung Dis 2011; 15: 1164-1169.

4 Becerra MC, Appleton SC, Franke MF, et al. Tuberculosis burden in households of patients with multidrug-resistant and extensively drug-resistant tuberculosis: a retrospective cohort study. Lancet 2011; 377: 147-152.

5 Annual reports 1997 and 2009, Tuberculosis and Chest Service, Department of Health, Hong Kong. Available from: www.chp. gov.hk/en/data/4/10/26/43/91 and www.info.gov.hk/tb_chest/ contents/c56.

6 World Health Organization. Anti-tuberculosis Drug Resistance in the World Report No. 4. The WHO/IUATLD global project on anti-tuberculosis drug resistance surveillance. Geneva, WHO press, 2008.

7 Law WS, Yew WW, Chiu Leung C, et al. Risk factors for multidrug-resistant tuberculosis in Hong Kong. Int J Tuberc Lung Dis 2008; 12: 1065-1070.

8 Noertjojo K, Tam CM, Chan SL, et al. Contact examination for tuberculosis in Hong Kong is useful. Int J Tuberc Lung Dis 2002; 6: $19-24$.

9 Leung CC, Tam CM, Chan SL, et al. Efficacy of the BCG revaccination programme in a cohort given BCG vaccination at birth in Hong Kong. Int J Tuberc Lung Dis 2001; 5: 717-723.

10 Leung ECC, Yew WW, Leung CC, et al. Shorter treatment duration for selected patients with multidrug-resistant tuberculosis. Eur Respir J 2011; 38: 227-230.

11 Hermans PW, van Soolingen D, Dale JW, et al. Insertion element IS986 from Mycobacterium tuberculosis: a useful tool for diagnosis and epidemiology of tuberculosis. J Clin Microbiol 1990; 28: 2051-2058.

12 Laserson KF, Thorpe LE, Leimane V, et al. Speaking the same language: treatment outcome definitions for multidrug-resistant tuberculosis. Int J Tuberc Lung Dis 2005; 9: 640-645.

13 Murray M, Alland D. Methodological problems in the molecular epidemiology of tuberculosis. Am J Epidemiol 2000; 155: 565-571.

14 Murray M, Nardell E. Molecular epidemiology of tuberculosis: achievements and challenges to current knowledge. Bull World Health Organ 2002; 80: 477-482.

15 Gandhi NR, Moll A, Sturm AW, et al. Extensively drug-resistant tuberculosis as a cause of death in patients co-infected with tuberculosis and HIV in a rural area of South Africa. Lancet 2006; 368: 1575-1580.

16 Zhao $\mathrm{M}, \mathrm{Li} \mathrm{X}, \mathrm{Xu} \mathrm{P}$, et al. Transmission of MDR and XDR tuberculosis in Shanghai, China. PLoS One 2009; 4: e4370.
17 Leimane V, Dravniece G, Riekstina V, et al. Treatment outcome of multidrug/extensively drug-resistant tuberculosis in Latvia, 20002004. Eur Respir J 2010; 36: 584-593.

18 Murase Y, Maeda S, Yamada H, et al. Clonal expansion of multidrug-resistant and extensively drug-resistant tuberculosis, Japan. Emerg Infect Dis 2010; 16: 948-954.

19 Chan-Yeung M, Kam KM, Leung CC, et al. Population based prospective molecular and conventional epidemiological study of tuberculosis in Hong Kong. Respirology 2006; 11: 442-448.

20 Devaux I, Kremer K, Heersma H, et al. Clusters of multidrugresistant Mycobacterium tuberculosis cases, Europe. Emerg Infect Dis 2009; 15: 1052-1060.

21 Kam KM, Yip CW, Tse LW, et al. Trends in multidrug-resistant Mycobacterium tuberculosis in relation to sputum smear positivity in Hong Kong, 1989-1999. Clin Infect Dis 2002; 34: 324-329.

22 Lee MS, Leung CC, Kam KM, et al. Early and late tuberculosis risks among close contacts in Hong Kong. Int J Tuberculosis Lung Dis 2008; 12: 281-287.

23 Grandjean L, Crossa A, Gilman RH, et al. Tuberculosis in household contacts of multidrug-resistant tuberculosis patients. Int J Tuberc Lung Dis 2011; 15: 1164-1169.

24 Morrison J, Pai M, Hopewell PC. Tuberculosis and latent tuberculosis infection in close contacts of people with pulmonary tuberculosis in low-income and middle-income countries: a systematic review and meta-analysis. Lancet Infect Dis 2008; 8: 359-368.

25 Dahle UR. Extensively drug resistant tuberculosis: beware patients lost to follow-up. BMJ 2006; 333: 705.

26 Dahle UR, Sandven P, Heldal E, et al. Deciphering an outbreak of drug resistant Mycobacterium tuberculosis. J Clin Microbiol 2003; 41: $67-72$.

27 Andersson DI. The biological cost of mutational antibiotic resistance: any practical conclusions? Curr Opin Microbiol 2006; 9: 461-465.

28 Metcalfe JZ, Kim EY, Lin SY, et al. Determinants of multidrugresistant tuberculosis clusters, California, USA, 2004-2007. Emerg Infect Dis 2010; 16: 1403-1409.

29 Lee JH, Ammerman NC, Nolan S, et al. Isoniazid resistance without a loss of fitness in Mycobacterium tuberculosis. Nat Commun 2012; 20: 753.

30 Comas I, Borrell S, Roetzer A, et al. Whole-genome sequencing of rifampicin-resistant Mycobacterium tuberculosis strains identifies compensatory mutations in RNA polymerase genes. Nat Genet 2011; 44: 106-110.

31 Dheda K, Migliori GB. The global rise of extensively drug-resistant tuberculosis: is the time to bring back sanatoria now overdue? Lancet 2012; 379: 773-775.

32 World Health Organization. Frequently asked questions - XDRTB. www.who.int/tb/challenges/xdr/faqs/en/index.html Date last accessed: $21 / 4 / 2012$. 